\title{
Analytical Approximate Solutions for a General Class of Nonlinear Delay Differential Equations
}

\author{
Bogdan Căruntu and Constantin Bota \\ Department of Mathematics, "Politehnica" University of Timişoara, Piaţa Victoriei 2, 300006 Timişoara, Romania \\ Correspondence should be addressed to Bogdan Căruntu; bogdan.caruntu@upt.ro
}

Received 12 June 2014; Accepted 9 July 2014; Published 24 July 2014

Academic Editor: Dumitru Baleanu

Copyright (C) 2014 B. Căruntu and C. Bota. This is an open access article distributed under the Creative Commons Attribution License, which permits unrestricted use, distribution, and reproduction in any medium, provided the original work is properly cited.

We use the polynomial least squares method (PLSM), which allows us to compute analytical approximate polynomial solutions for a very general class of strongly nonlinear delay differential equations. The method is tested by computing approximate solutions for several applications including the pantograph equations and a nonlinear time-delay model from biology. The accuracy of the method is illustrated by a comparison with approximate solutions previously computed using other methods.

\section{Introduction}

Delay differential equations are frequently used to model reallife phenomena in various fields such as mechanics, biology, computer science, and chemistry. Some of the recent studies involving delay differential equations include topics as varied as epidemic models that describe the fraction of a population infected by a virus [1], complex oscillator network [2], and neural networks [3].

It is known that the computation of exact solutions for delay differential equations is only possible in particular cases. It follows that, in most cases, in order to obtain information about the phenomena modeled, the computation of approximate solutions becomes a necessity.

In the present paper we used the polynomial least squares method (PLSM) to compute approximate solutions for the following class of nonlinear delay differential equations:

$$
\begin{aligned}
& F\left(x^{(n)}\left(h_{n}(t)\right), x^{(n-1)}\left(h_{n-1}(t)\right),\right. \\
& \left.\quad x^{(n-2)}\left(h_{n-2}(t)\right), \ldots, x^{(1)}\left(h_{1}(t)\right), x\left(h_{0}(t)\right), t\right)=0,
\end{aligned}
$$

together with the initial conditions

$$
\sum_{i=0}^{n-1} r_{i j} x^{(i)}(\alpha)=s_{j}, \quad j \in 1,2, \ldots, n
$$

Here $F$ is a function which satisfies such conditions as necessary to ensure that the problem (1)-(2) admits a unique solution, $r_{i j}$ and $s_{j}$ are real constants, and the functions $h_{k}(t)$, $k \in 0,1, \ldots, n$, are polynomial functions in the $t$ variable, $t \in[\alpha, \beta]$.

Among the methods recently used to compute approximate solutions for various delay differential equations of (1) type we mention the following.

(i) In [4] a numerical approximation based on the Bessel functions of the first kind was applied to (1) of the Riccati type.

(ii) In [5] a two-stage order-one Runge-Kutta method was applied to (1) of the neutral-function differential type. The same equation was studied in $[6,7]$ by using the one-leg $\theta$-method, in [8] by using the variational iteration method, in [9] by using the homotopy perturbation method, and in [10] by using a method based on Chebyshev polynomials.

(iii) In [11] the homotopy perturbation method was applied to (1) of the pantograph type. The same equation was studied in [12] by using the variational iteration method.

(iv) In [13] the Jacobi rational-Gauss collocation method was applied to (1) of the generalized pantograph type. The same equation was studied in [14] by using the 
Taylor series method, in [15] by using a Chebyshev method, and in [16] by using a Hermite collocation method.

(v) In [17] the variational iteration method and the Adomian decomposition method were applied for the case of a nonlinear time-delay model in biology which is also a particular case of (1).

(vi) In $[18,19]$ local fractional methods (local fractional variation iteration method and local fractional Adomian decomposition method, resp.) were applied.

In the next section we introduce the polynomial least squares method (PLSM), which allows us to find analytical approximate polynomial solutions for the problem (1)-(2), and in the third section we compare our approximate solutions with approximate solutions presented in [4-17].

\section{The Polynomial Least Squares Method}

Let $D$ be the operator associated with the differential equation (1):

$$
\begin{aligned}
D(x)=F\left(x^{(n)}\left(h_{n}(t)\right), x^{(n-1)}\left(h_{n-1}(t)\right),\right. \\
\left.\quad x^{(n-2)}\left(h_{n-2}(t)\right), \ldots, x^{(1)}\left(h_{1}(t)\right), x\left(h_{0}(t)\right), t\right) .
\end{aligned}
$$

The error obtained by replacing the exact solution $x$ with an approximate solution $x_{\alpha}$ is given by the so-called remainder:

$$
R\left(t, x_{\alpha}\right)=D\left(x_{\alpha}(t)\right), \quad t \in[\alpha, \beta] .
$$

As a consequence, we will search for approximate polynomial solutions $x_{\alpha}$ of (1)-(2) on the [ $\left.\alpha, \beta\right]$ interval, solutions which satisfy the following conditions:

$$
\begin{gathered}
\left|R\left(t, x_{\alpha}\right)\right|<\epsilon, \quad \in \in \mathbb{R}_{+}, \\
\sum_{i=0}^{n-1} r_{i j} x^{(i)}(\alpha)=s_{j}, \quad j \in 1,2, \ldots, n .
\end{gathered}
$$

Definition 1. (a) We call an $\epsilon$-approximate polynomial solution of the problem (1)-(2) an approximate polynomial solution $x_{\alpha}$ satisfying the relations (5) and (6).

(b) We call a weak $\delta$-approximate polynomial solution of the problem (1)-(2) an approximate polynomial solution $x_{\alpha}$ satisfying the relation

$$
\int_{\alpha}^{\beta} R^{2}\left(t, x_{\alpha}\right) d t \leq \delta, \quad \delta \in \mathbb{R}_{+},
$$

together with the initial condition (6).

Remark 2. Taking into account the way the problem (1)-(2) is defined, from the Weierstrass approximation theorem it follows that there exists the sequence of polynomials, $P_{m}(t)=$ $\beta_{0}+\beta_{1} t+\cdots+\beta_{m} t^{m}, \beta_{i} \in \mathbb{R}, i=0,1, \ldots, m$, satisfying the conditions

$$
\sum_{i=0}^{n-1} r_{i j} P_{m}^{(i)}(\alpha)=s_{j}, \quad j \in 1,2, \ldots, n,
$$

such that the sequence of polynomials $P_{m}(t)$ is convergent to the solution of the problem (1)-(2); that is, $\lim _{m \rightarrow \infty} D\left(P_{m}(t)\right)=$ 0 .

Remark 3. Any $\epsilon$-approximate polynomial solution of the problem (1)-(2) is also a weak $\epsilon^{2} \cdot(\beta-\alpha)$-approximate polynomial solution, but the opposite is not always true. It follows that the set of weak approximate solutions of the problem (1)-(2) also contains the approximate solutions of the problem.

Theorem 4. The problem (1)-(2) admits a sequence of weak approximate polynomial solutions.

Proof. Taking into account the definition, we will find a weak $\epsilon$-polynomial solution of the type

$$
\tilde{x}(t)=\sum_{k=0}^{m} c_{k} \cdot t^{k}, \quad m>n,
$$

where the constants $c_{0}, c_{1}, \ldots, c_{m}$ are calculated using the following steps.

(1) In the first step we substitute the approximate solution (9) in (1) and obtain the remainder:

$$
\begin{aligned}
& \Re\left(t, c_{0}, c_{1}, \ldots, c_{m}\right) \\
& =R(t, \tilde{x})=F\left(\tilde{x}^{(n)}\left(h_{n}(t)\right), \tilde{x}^{(n-1)}\left(h_{n-1}(t)\right), \tilde{x}^{(n-2)}\right. \\
& \left.\quad \times\left(h_{n-2}(t)\right), \ldots, \tilde{x}^{(1)}\left(h_{1}(t)\right), \tilde{x}\left(h_{0}(t)\right), t\right) .
\end{aligned}
$$

(2) Next we compute $c_{0}, c_{1}, \ldots, c_{n-1}$ as functions of $c_{n}, \ldots, c_{m}$ by using the initial conditions:

$$
\sum_{i=0}^{n-1} r_{i j} \tilde{x}^{(i)}(\alpha)=s_{j}, \quad j \in 1,2, \ldots, n \text {. }
$$

(3) We attach to the problem (1)-(2) the following real functional:

$$
J\left(c_{n}, \ldots, c_{m}\right)=\int_{\alpha}^{\beta} \mathfrak{R}^{2}\left(t, c_{0}, c_{1}, \ldots, c_{m}\right) d t .
$$

(4) Next we compute the values of $c_{n}^{0}, \ldots, c_{m}^{0}$ as the values which give the minimum of the functional (12) and the values of $c_{0}^{0}, c_{1}^{0}, \ldots, c_{n-1}^{0}$ again as functions of $c_{n}^{0}, \ldots, c_{m}^{0}$ by using the initial conditions.

(5) By using the constants $c_{0}^{0}, c_{1}^{0}, \ldots, c_{m}^{0}$ thus determined, we consider the polynomial:

$$
T_{m}(t)=\sum_{k=0}^{m} c_{k}^{0} \cdot t^{k}
$$

Based on the way the coefficients of polynomial $T_{m}(t)$ are computed and taking into account the relations (10)-(13), the following inequality holds:

$$
0 \leq \int_{\alpha}^{\beta} R^{2}\left(t, T_{m}(t)\right) d t \leq \int_{\alpha}^{\beta} R^{2}\left(t, P_{m}(t)\right) d t, \quad \forall m \in \mathbb{N} .
$$


It follows that

$$
0 \leq \lim _{m \rightarrow \infty} \int_{\alpha}^{\beta} R^{2}\left(t, T_{m}(t)\right) d t \leq \lim _{m \rightarrow \infty} \int_{\alpha}^{\beta} R^{2}\left(t, P_{m}(t)\right) d t=0 .
$$

We obtain

$$
\lim _{m \rightarrow \infty} \int_{\alpha}^{\beta} R^{2}\left(t, T_{m}(t)\right) d t=0 .
$$

From this limit we obtain that $\forall \epsilon>0, \exists m_{0} \in \mathbb{N}$ such that $\forall m \in \mathbb{N}, m>m_{0}$. It follows that $T_{m}(t)$ is a weak $\epsilon$ approximate polynomial solution of the problem (1)-(2).

As a consequence of the second remark, in order to find $\epsilon$-approximate polynomial solutions of the problem (1)-(2) by PLSM, we will first determine weak approximate polynomial solutions, $\tilde{x}_{\alpha}$ following the steps 1 to 5 previously described. If $\left|R\left(t, \tilde{x}_{\alpha}\right)\right|<\epsilon$, then $\tilde{x}_{\alpha}$ is also an $\epsilon$-approximate polynomial solution of the problem.

\section{Applications}

3.1. Application 1: Nonlinear Riccati Equation. Our first test problem is the following Cauchy problem:

$$
\begin{gathered}
x^{\prime}(t-2)-t^{2} \cdot x(2 \cdot t-3)-x^{2}(t-1)+5 t^{4}-20 t^{3} \\
+19 t^{2}-2 t-3=0, \\
x(0)=-2 .
\end{gathered}
$$

The exact solution of this problem is $x_{e}(t)=t^{2}-t-2$.

In [4], by using a numerical approximation based on the Bessel functions of the first kind, Yüzbaşi computed the following approximate solution of (17):

$$
x_{\mathrm{BES}}=\left(0.1 \cdot 10^{-19}\right) t^{3}+t^{2}-1.0000000000000000001 t-2 .
$$

The maximum absolute error of this approximation is reported as $1.2171 \cdot 10^{-19}$.

Using the steps described in the previous section we performed the following computations.

(i) We computed a polynomial solution of the form

$$
x_{\mathrm{PLSM}}=c_{0}+c_{1} \cdot t+c_{2} \cdot t^{2} .
$$

(ii) Taking into account the fact that, by using the initial condition, $c_{0}$ must be equal to -2 , the functional (12) corresponding to the problem (17) is

$$
\begin{aligned}
J\left(c_{1}, c_{2}\right)= & \frac{c_{1}^{4}}{5}-\frac{2 c_{1}^{3} c_{2}}{3}+\frac{6 c_{1}^{3}}{5}+\frac{6 c_{1}^{2} c_{2}^{2}}{7}-\frac{134 c_{1}^{2} c_{2}}{21} \\
& +\frac{701 c_{1}^{2}}{105}-\frac{c_{1} c_{2}^{3}}{2}+\frac{107 c_{1} c_{2}^{2}}{14}-\frac{1049 c_{1} c_{2}}{70}+\frac{1963 c_{1}}{210} \\
& +\frac{c_{2}^{4}}{9}-\frac{25 c_{2}^{3}}{9}+\frac{1206 c_{2}^{2}}{35}-\frac{18341 c_{2}}{315}+\frac{8543}{315}
\end{aligned}
$$

(iii) To find the minimum of this functional we compute the stationary points as the solutions of the system

$$
\begin{gathered}
\frac{\partial J}{\partial c_{1}}=0, \\
\frac{\partial J}{\partial c_{2}}=0 .
\end{gathered}
$$

Since the only stationary point is $c_{1}=-1, c_{2}=$ 1 and it is easy to show that this point is indeed a minimum, we obtain the following polynomial approximate solution of (17):

$$
x_{\mathrm{PLSM}}=-2-t+t^{2},
$$

which is actually the exact solution of the problem.

We remark that while in this simple case we were able to compute the exact minimum of the functional (12) in most of the applications the direct computation of the minimum is not possible and some numerical techniques are employed.

3.2. Application 2: Second-Order Neutral Functional-Differential Equation with Proportional Delays. Our second test problem is the following Cauchy problem:

$$
\begin{gathered}
x^{\prime \prime}(t)-\frac{3}{4} x(t)-x\left(\frac{t}{2}\right)-x^{\prime}\left(\frac{t}{2}\right)-\frac{1}{2} x^{\prime \prime}\left(\frac{t}{2}\right) \\
+t^{2}+t-1=0, \quad 0 \leq t \leq 1, \\
x(0)=0, \quad x^{\prime}(0)=0 .
\end{gathered}
$$

The exact solution of this problem is $x_{e}(t)=t^{2}$.

In [5], Bellen and Zennaro used a two-stage order-one Runge-Kutta method to compute a numerical solution of the problem (23) and the absolute error of their approximation is of the order $10^{-3}$. In $[6,7]$, Wang et al. used the one-leg $\theta$-method to compute approximate solutions of (23) and the absolute error of their best approximation is of the order $10^{-3}$. In [8], Chen and Wang used the variational iteration method to compute approximate solutions of (23) and the absolute error of their best approximation is of the order $10^{-6}$. In [9], Biazar and Ghanbari used the homotopy perturbation method (HPM) to compute approximate solutions of (23) and the absolute error of their best approximation is of the order $10^{-6}$. In [10], Sedaghat et al. used a method based on Chebyshev polynomials to compute a numerical solution of the problem (23) and the absolute error of their approximation is of the order $10^{-17}$. tions.

Using our method we performed the following computa-

(i) We compute a polynomial solution of the form

$$
x_{\mathrm{PLSM}}=c_{0}+c_{1} \cdot t+c_{2} \cdot t^{2} .
$$

(ii) Taking into account the initial conditions we obtain the following values of the constants $c_{0}=0, c_{1}=0$. 
TABLE 1: Comparison of VIM, HPM, and PLSM for (27).

\begin{tabular}{lccc}
\hline$t$ & VIM & HPM & PLSM \\
\hline 0.2 & $5 \cdot 10^{-5}$ & $1.3410^{-5}$ & $1.0210^{-8}$ \\
0.4 & $1 \cdot 10^{-4}$ & $5.1310^{-5}$ & $6.1410^{-8}$ \\
0.6 & $5 \cdot 10^{-5}$ & $6.2610^{-6}$ & $8.9210^{-8}$ \\
0.8 & $1 \cdot 10^{-4}$ & $2.2110^{-5}$ & $1.0210^{-7}$ \\
1 & $5 \cdot 10^{-4}$ & $3.6910^{-5}$ & $1.5310^{-7}$ \\
\hline
\end{tabular}

In this case the functional (12) corresponding to the problem (23) is

$$
J\left(c_{2}\right)=\frac{11 c_{2}^{2}}{30}-\frac{11 c_{2}}{15}+\frac{11}{30} .
$$

(iii) To find the minimum of this functional we compute the stationary points as the solutions of the equation $J^{\prime}\left(c_{2}\right)=0$. The only stationary point is $c_{2}=1$ and it is easy to show that this point is indeed a minimum.

(iv) We obtain the following polynomial approximate solution of (23):

$$
x_{\mathrm{PLSM}}=t^{2} \text {. }
$$

Again we obtained the exact solution of the problem.

3.3. Application 3: Pantograph-Type Nonlinear Equation. Our third test problem is the following Cauchy problem:

$$
\begin{gathered}
x^{\prime \prime}(t)-e^{-t} x^{\prime}\left(t-\frac{1}{5}\right)-(t-1) x(t)+2 x\left(\frac{t}{3}\right)-x^{2}(t) \\
-\left(-\frac{1}{4} \sin ^{2}\left(\frac{t}{3}\right)+\frac{4}{9} \sin \left(\frac{t}{3}\right)+\sin \left(\frac{t}{9}\right)-\exp (-t)\right. \\
\quad \times\left(\frac{1}{6} \cos \left(\frac{t}{3}-\frac{1}{15}\right)-\frac{1}{6} \sin \left(\frac{t}{2}-\frac{1}{10}\right)\right)-\frac{1}{9} \cos ^{2}\left(\frac{t}{2}\right) \\
+\frac{1}{4} \cos \left(\frac{t}{2}\right)+\frac{2}{3} \cos \left(\frac{t}{6}\right)-\frac{1}{3} \sin \left(\frac{t}{3}\right) \cos \left(\frac{t}{2}\right) \\
\left.+t\left(-\frac{1}{2} \sin \left(\frac{t}{3}\right)-\frac{1}{3} \cos \left(\frac{t}{2}\right)\right)\right)=0, \\
3 x(0)+6 x^{\prime}(0)=2, \\
-2 x(0)+x^{\prime}(0)=-\frac{1}{2} .
\end{gathered}
$$

The exact solution of this problem is $x_{e}(t)=(1 / 2) \sin (t /$ $3)+(1 / 3) \cos (t / 2)$.

In [11], Shakeri and Dehghan used the homotopy perturbation method (HPM) to compute approximate solutions $x_{\mathrm{HPM}}$ of (27).

In [12], Yildirim et al. used the variational iteration method (VIM) to compute approximate solutions $x_{\text {VIM }}$ of (27).
Using our method we obtained the following polynomial approximate solution of (27):

$$
\begin{aligned}
x_{\mathrm{PLSM}}= & -3.8337691 \cdot 10^{-6} \cdot t^{5}+0.00088928 \cdot t^{4} \\
& -0.00309479 \cdot t^{3}-0.0416659 \cdot t^{2}+\frac{1}{6} \cdot t+\frac{1}{3} .
\end{aligned}
$$

Table 1 presents the comparison between the absolute errors (as the difference in absolute value between the approximate solution and the exact solution) corresponding to the approximate solution $x_{\mathrm{HPM}}$ from [11], to the approximate solution $x_{\text {VIM }}$ from [12], and to our approximate solution $x_{\text {PLSM }}$. The absolute errors corresponding to the approximate solution $x_{\mathrm{VIM}}$ are not explicitly given in [12], but we extracted some approximate values from a figure included in [12] (namely, Figure 2(b)).

It is easy to see that the approximate solution given by PLSM is much closer to the exact solution than the previous ones from $[11,12]$. We mention the fact that our solution not only is more precise but also, at the same time, has a much simpler form.

3.4. Application 4: Generalized Pantograph-Type Equation. Our third test problem is the following Cauchy problem:

$$
x^{\prime \prime \prime}(t)+x(t)+x(t-0.3)-e^{-t+0.3},
$$

$x(0)=1, \quad x^{\prime}(0)=-1, \quad x^{\prime \prime}(0)=1, \quad 0 \leq t \leq 1$.

The exact solution of this problem is $x_{e}(t)=e^{-t}$.

In [13], Doha et al. used the Jacobi rational-Gauss collocation method (JRC) to compute approximate solutions $x_{\text {IRC }}$ of (29). In [14], Sezer and Akyuz-Dascioglu used the Taylor series method (TM) to compute approximate solutions $x_{\mathrm{TM}}$ of (29). In [15], Ozturk and Gulsu used a Chebyshev method (CM) to compute approximate solutions $x_{\mathrm{CM}}$ of (29). In [16], Yalçinbaş et al. used a Hermite collocation method (HCM) to compute approximate solutions $x_{\mathrm{HCM}}$ of the same equation.

Using our method we obtained the following polynomial approximate solution of (29):

$$
\begin{aligned}
& x_{\mathrm{PLSM}} \\
&=-1.6853299 \cdot 10^{-6} \cdot t^{9}+0.0000227752 \cdot t^{8} \\
&-0.000196271 \cdot t^{7}+0.00138757 \cdot t^{6}-0.00833288 \cdot t^{5} \\
&+0.0416666 \cdot t^{4}-0.166667 \cdot t^{3}+\frac{t^{2}}{2}-t+1 .
\end{aligned}
$$

Table 2 presents the comparison between the absolute errors (as the difference in absolute value between the approximate solution and the exact solution) corresponding to the approximate solution $x_{\mathrm{TM}}$ from [14], to the approximate solution $x_{\mathrm{CM}}$ from [15], to the approximate solution $x_{\mathrm{HCM}}$ from [16], and to the (best) approximate solution $x_{\text {JRC }}$ from [13], as given in [13], together with the errors corresponding to our approximate solution $x_{\text {PLSM }}$. 
TABLE 2: Comparison of TM, CM, HCM, JRC, and PLSM for (29).

\begin{tabular}{lccccc}
\hline$t$ & TM & CM & HCM & JRC & 0 \\
\hline 0 & 0 & 0 & 0 & $3.60510^{-8}$ & 0 \\
0.2 & $8.54 \cdot 10^{-8}$ & $3.7010^{-7}$ & $6.20010^{-9}$ & $9.29910^{-9}$ & $6.25510^{-13}$ \\
0.4 & $5.36 \cdot 10^{-6}$ & $2.3810^{-6}$ & $5.76010^{-8}$ & $3.50310^{-10}$ & $7.19310^{-12}$ \\
0.6 & $5.95 \cdot 10^{-5}$ & $5.9710^{-6}$ & $1.79610^{-7}$ & $8.34510^{-9}$ & $1.83910^{-11}$ \\
0.8 & $3.26 \cdot 10^{-4}$ & $3.4810^{-5}$ & $3.73510^{-7}$ & $1.16110^{-8}$ & $3.34110^{-11}$ \\
1 & $1.21 \cdot 10^{-3}$ & $2.0310^{-4}$ & $6.36810^{-7}$ & $5.64210^{-11}$ \\
\hline
\end{tabular}

TABle 3: Comparison of VIM, HPM, and PLSM for (31).

\begin{tabular}{lccc}
\hline$t$ & ADM & VIM & PLSM \\
\hline 0 & 0 & 0 & 0 \\
0.05 & $1.04 \cdot 10^{-1}$ & - & $6.3310^{-2}$ \\
0.1 & $1.38 \cdot 10^{-1}$ & - & $7.8610^{-2}$ \\
0.15 & $1.31 \cdot 10^{-1}$ & - & $7.1310^{-2}$ \\
0.2 & $1.26 \cdot 10^{-1}$ & - & $5.8310^{-2}$ \\
0.25 & $1.25 \cdot 10^{-1}$ & - & $4.4710^{-2}$ \\
0.3 & $1.26 \cdot 10^{-1}$ & - & $3.2710^{-2}$ \\
0.35 & $1.27 \cdot 10^{-1}$ & - & $2.3210^{-2}$ \\
0.4 & $1.29 \cdot 10^{-1}$ & - & $1.6610^{-2}$ \\
0.5 & - & $1.5910^{-2}$ & $1.0710^{-2}$ \\
1 & - & $4.310^{-3}$ & $7.4110^{-4}$ \\
1.5 & - & $8.210^{-4}$ & $6.1410^{-4}$ \\
\hline
\end{tabular}

Again it is easy to see that the approximate solution given by PLSM is much closer to the exact solution than the previous ones.

3.5. Application 5: Nonlinear Time-Delay Model in Biology. Our next test problem is

$$
\begin{gathered}
x^{\prime}(t)-2 \cdot x(t)\left(1-\frac{x(t-0.1)}{0.5}\right)=0, \\
x(0)=1 .
\end{gathered}
$$

The exact solution of this problem is not known.

In [17], Dehghan and Salehi used the variational iteration method (VIM) and the Adomian decomposition method $(\mathrm{ADM})$ to compute approximate solutions $x_{\mathrm{VIM}}$ and $x_{\mathrm{ADM}}$ of (31).

Using our method we obtained the following polynomial approximate solution of (31):

$$
\begin{aligned}
& x_{\mathrm{PLSM}} \\
&=-2.57841 \cdot t^{7}+15.8186 \cdot t^{6}-39.5946 \cdot t^{5}+52.059 \cdot t^{4} \\
&-38.6665 \cdot t^{3}+16.3752 \cdot t^{2}-3.89839 \cdot t+1 .
\end{aligned}
$$

Table 3 presents the comparison between the absolute errors (as the difference in absolute value between the approximate solution and the numerical solution presented in [17]) corresponding to the approximate solutions $x_{\mathrm{ADM}}$ and $x_{\mathrm{VIM}}$ from [17] and to our approximate solution $x_{\mathrm{PLSM}}$.
The approximate solution given by PLSM is closer to the numerical solution than the previous ones from [17].

\subsection{Application 6: Scalar Differential Equation with Several} Delays. Our last test problem is

$$
\begin{gathered}
x^{\prime}(t)+\frac{0.2}{\pi} x(t-\pi) \sin ^{2}(t)+\frac{0.2}{\pi} x(t-2 \pi) \cos ^{2}(t)=0, \\
x(0)=1 .
\end{gathered}
$$

The exact solution of this problem is not known. In [20], Berezansky and Braverman studied the existence of positive solutions for equations of the type

$$
x^{\prime}(t)+\sum_{k=0}^{m} \alpha_{k}(t) x\left(h_{k}(t)\right)=0 .
$$

In the case of (33) it was shown that there exists indeed such a positive solution, but the solution was not effectively computed.

Using our method on the interval $[0,1]$ we obtained the following polynomial approximate solution of (33):

$$
\begin{aligned}
& x_{\mathrm{PLSM}} \\
&=-7.0502 \cdot 10^{-6} \cdot t^{5}-0.0000361911 \cdot t^{4}+0.000175863 \cdot t^{3} \\
&+0.000388792 \cdot t^{2}-0.00895158 \cdot t+0.1 .
\end{aligned}
$$

The error obtained by replacing the approximate solution back in the equation and computing the remainder is of the order $10^{-7}$.

It is easy to see that the solution is positive on the interval, where the computation was performed.

\section{Conclusions}

The polynomial least squares method (PLSM) was presented as a straightforward and efficient method to compute approximate polynomial solutions for nonlinear delay differential equations.

The applications presented clearly illustrate the accuracy of the method. Indeed, for the equations of the type (1) considered, namely, (17)-(33), the solutions obtained by using PLSM are more precise than the ones previously computed by using other methods. Moreover, for some problems PLSM was able to compute the exact solution while the other methods only produced approximate ones. 


\section{Conflict of Interests}

The authors declare that there is no conflict of interests regarding the publication of this paper.

\section{References}

[1] C. Zhang and H. Chen, "Block boundary value methods for delay differential equations," Applied Numerical Mathematics. An IMACS Journal, vol. 60, no. 9, pp. 915-923, 2010.

[2] Y. Li and W. Jiang, "Nonlinear waves in complex oscillator network with delay," Communications in Nonlinear Science and Numerical Simulation, vol. 18, no. 11, pp. 3226-3237, 2013.

[3] F. Zhang and Y. Zhang, "State estimation of neural networks with both time-varying delays and norm-bounded parameter uncertainties via a delay decomposition approach," Communications in Nonlinear Science and Numerical Simulation, vol. 18, no. 12, pp. 3517-3529, 2013.

[4] Ş. Yüzbaşı, "A numerical approximation based on the Bessel functions of first kind for solutions of Riccati type differentialdifference equations," Computers \& Mathematics with Applications, vol. 64, no. 6, pp. 1691-1705, 2012.

[5] A. Bellen and M. Zennaro, Numerical Methods for Delay Differential Equations. Numerical Mathematics and Scientific Computation, The Clarendon Press Oxford University Press, New York, NY, USA, 2003.

[6] W. Wang and S. Li, "On the one-leg $\theta$-methods for solving nonlinear neutral functional differential equations," Applied Mathematics and Computation, vol. 193, no. 1, pp. 285-301, 2007.

[7] W. Wang, T. Qin, and S. Li, "Stability of one-leg $\theta$-methods for nonlinear neutral differential equations with proportional delay," Applied Mathematics and Computation, vol. 213, no. 1, pp. 177-183, 2009.

[8] X. Chen and L. Wang, "The variational iteration method for solving a neutral functional-differential equation with proportional delays," Computers \& Mathematics with Applications, vol. 59, no. 8, pp. 2696-2702, 2010.

[9] J. Biazar and B. Ghanbari, “The homotopy perturbation method for solving neutral functional-differential equations with proportional delays," Journal of King Saud University-Science, vol. 24, no. 1, pp. 33-37, 2012.

[10] S. Sedaghat, Y. Ordokhani, and M. Dehghan, "Numerical solution of the delay differential equations of pantograph type via Chebyshev polynomials," Communications in Nonlinear Science and Numerical Simulation, vol. 17, no. 12, pp. 4815-4830, 2012.

[11] F. Shakeri and M. Dehghan, "Solution of delay differential equations via a homotopy perturbation method," Mathematical and Computer Modelling, vol. 48, no. 3-4, pp. 486-498, 2008.

[12] A. Yildirim, H. Koçak, and S. Tutkun, "Reliable analysis for delay differential equations arising in mathematical biology," Journal of King Saud University -Science, vol. 24, no. 4, pp. 359365, 2012.

[13] E. H. Doha, A. H. Bhrawy, D. Baleanu, and R. M. Hafez, "A new Jacobi rational-Gauss collocation method for numerical solution of generalized pantograph equations," Applied Numerical Mathematics, vol. 77, pp. 43-54, 2014.

[14] M. Sezer and A. Akyuz-Dascioglu, "A Taylor method for numerical solution of generalized pantograph equations with linear functional argument," Journal of Computational and Applied Mathematics, vol. 200, no. 1, pp. 217-225, 2007.
[15] Y. Ozturk and M. Gulsu, "Approximate solution of linear generalized pantograph equations with variable coefficients on Chebyshev-Gauss grid," Journal of Advanced Research in Scientific Computing, vol. 4, no. 1, pp. 36-51, 2012.

[16] S. Yalçinbaş, M. Aynigül, and M. Sezer, "A collocation method using Hermite polynomials for approximate solution of pantograph equations," Journal of The Franklin Institute, vol. 348, no. 6, pp. 1128-1139, 2011.

[17] M. Dehghan and R. Salehi, "Solution of a nonlinear time-delay model in biology via semi-analytical approaches," Computer Physics Communications, vol. 181, no. 7, pp. 1255-1265, 2010.

[18] X.-J. Yang and D. Baleanu, "Fractal heat conduction problem solved by local fractional variation iteration method," Thermal Science, vol. 17, no. 2, pp. 625-628, 2013.

[19] X.-J. Yang, D. Baleanu, and W.-P. Zhong, "Approximate solutions for diffusion equations on Cantor space-time," Proceedings of the Romanian Academy A, vol. 14, no. 2, pp. 127-133, 2013.

[20] L. Berezansky and E. Braverman, "Positive solutions for a scalar differential equation with several delays," Applied Mathematics Letters, vol. 21, no. 6, pp. 636-640, 2008. 


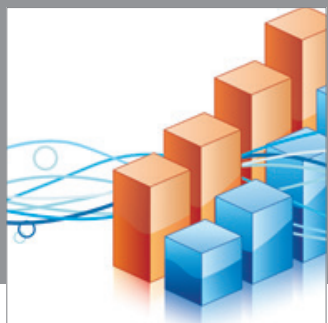

Advances in

Operations Research

mansans

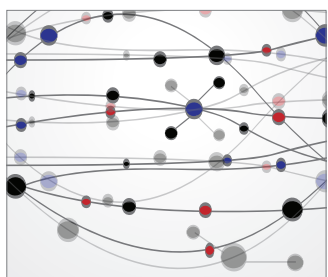

The Scientific World Journal
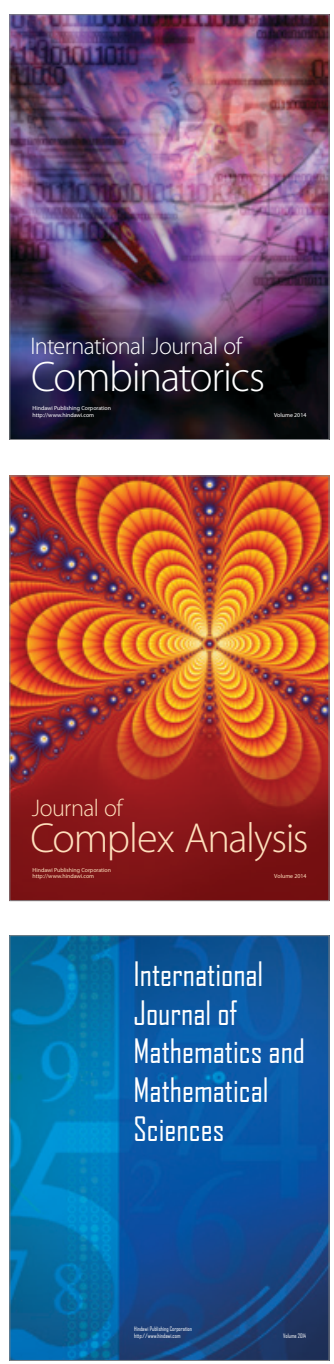
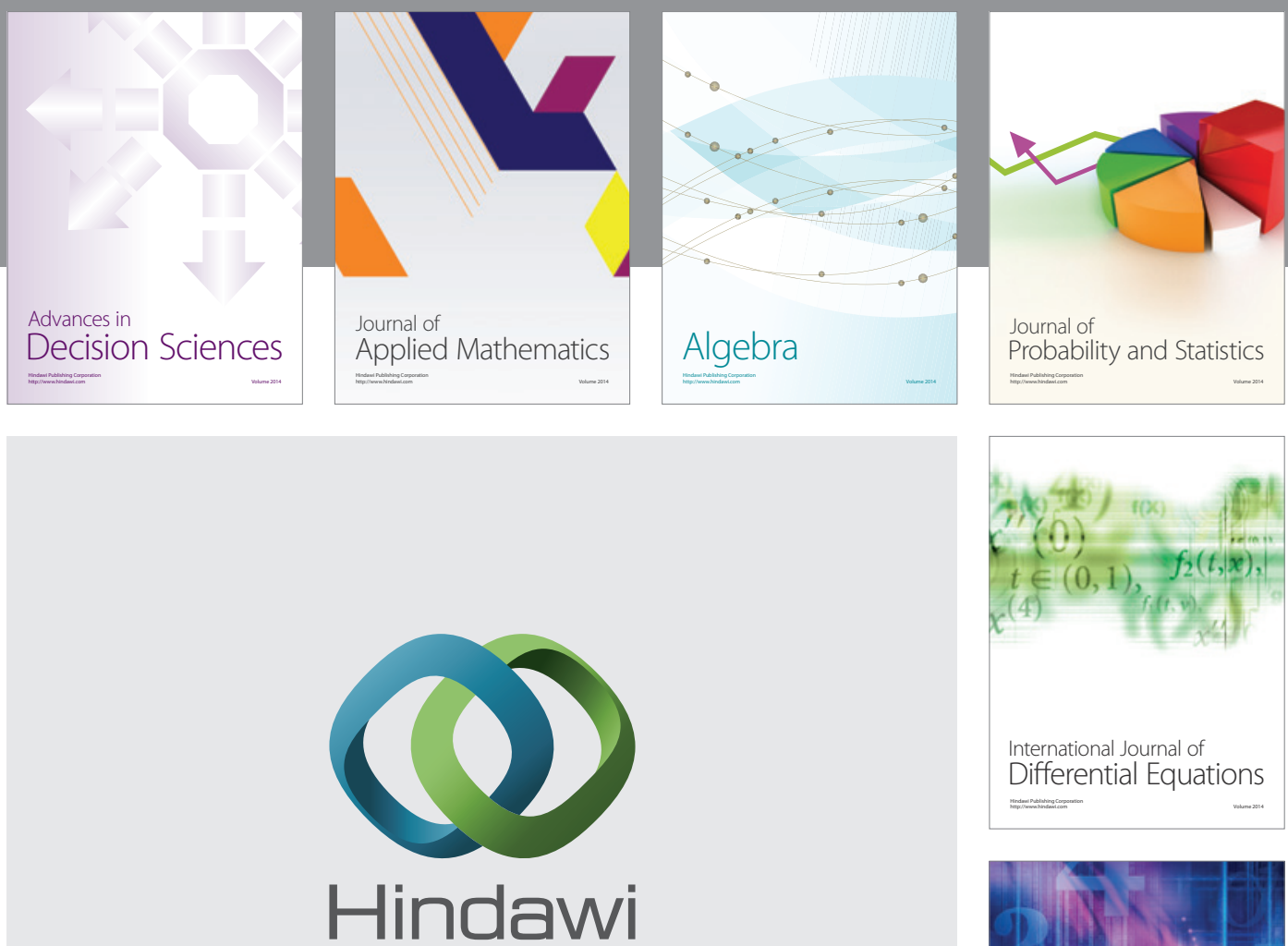

Submit your manuscripts at http://www.hindawi.com
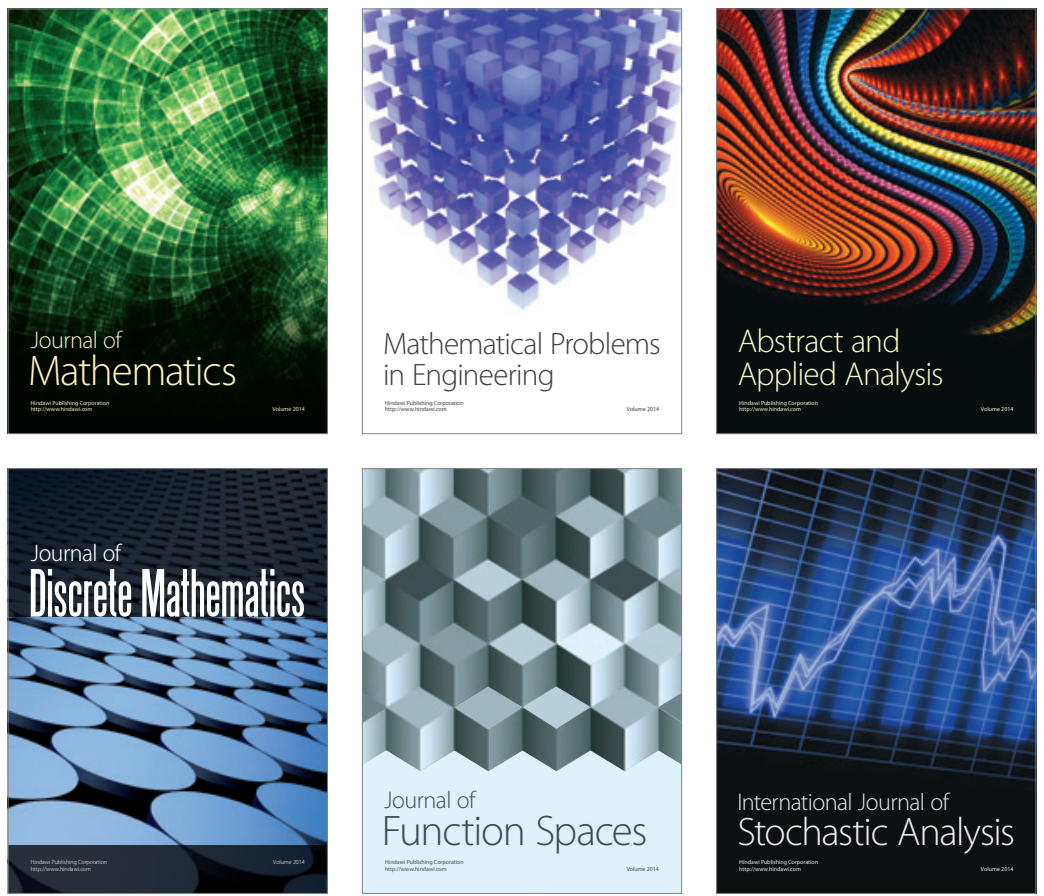

Journal of

Function Spaces

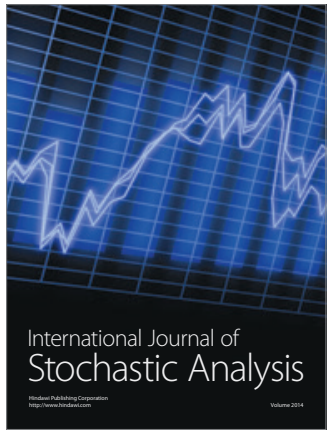

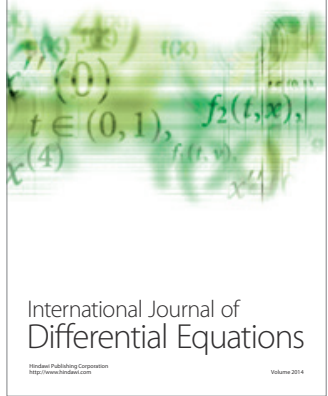
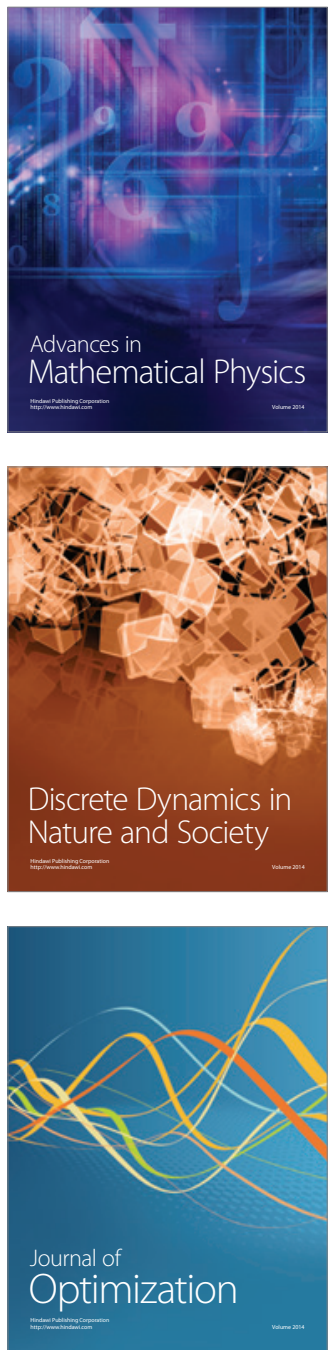